some 200,000 years ago, Homo sapiens.

Baggott ends at consciousness, that frontier of interdisciplinarity. But he fails to ask whether intelligent life is a convergent property of evolution. Given that evolution involves dominating local resources, the Universe may teem with 'dumb' life, while intelligent life remains exceedingly rare.

Earth's prehistory was marked by five major extinctions, identified in 1982 by palaeontologists David Raup and John Sepkoski. The Cretaceous-Palaeogene extinction 66 million years ago, which killed the dinosaurs, is the best known. Physicist Luis Alvarez and his geologist son Walter proposed that the cause was an asteroid impact, an idea met with scepticism until the mid-1990s, after a crater fitting the bill was identified in Mexico. Such an impact can alter conditions on Earth for tens of years, through a global dust cloud, firestorms and other after-effects: species ill-suited to such dramatic change go extinct.

Raup and Sepkoski also put forth evidence that extinction events occur roughly every 30 million years. This is now generally accepted, but there is no agreed mechanism. Randall and her collaborator Matt Reece offer a hypothesis. They posit that there are two kinds of dark matter: the ordinary one, whose gravity binds galaxies and galaxy clusters, and a 'social' form that also interacts with its own kind. The social dark matter forms a thin disk of material in our Galaxy whose gravity can shake things loose in the outer depths of the Solar System when it crosses the Galactic disk, every 30 million years or so. Randall admits that the idea is a long shot, although testable. This aspect of Dark Matter and the Dinosaurs conveys the excitement and uncertainty of cutting-edge, big-idea research.

In a chapter called 'The cosmic imperative', Baggott implies that the evolution of life is an inevitable consequence of chemistry, despite our not knowing precisely how it occurred. This reminded me of physicist Murray Gell-Mann's dictum "Everything not forbidden is compulsory" (borrowed from novelist T. H. White), which describes the importance of symmetry principles in particle physics: they set the basic rules, but not the detailed outcomes. A rich set of rules (think chess) can lead to complex and interesting outcomes. I would take this further: the Universe is governed by physical laws that permit a rich set of behaviours, resulting in its inevitable evolution from vacuum energy to quark soup, nuclei and atoms, all the way to the emergence of life and selfawareness. But that does not explain where space, time and the laws came from, or why there is something rather than nothing.

I have quibbles with Baggott's book. $\mathrm{He}$ gives a dated picture of inflation (tying it to symmetry breaking), gets the temperature of the cosmic microwave background wrong (it is 2.7255 kelvin) and calls the lumpiness that led to the formation of cosmic structures anisotropy, rather than inhomogeneity. But these gaffes do not interfere with the larger narrative and are a by-product of his sweeping scope and detailed description.

The longing to understand our place in the cosmos is universal. Baggott and Randall lay out how much of the story we understand, and how interconnected it all is. They remind us that big questions remain in this most wonderful scientific adventure.

Michael S. Turner is professor of astronomy and astrophysics, and of physics at the University of Chicago, Illinois, and director of the Kavli Institute for Cosmological Physics. e-mail:mturner@kicp.uchicago.edu

\title{
The mechanics of mind
}

\section{Daniel Bor enjoys a sophisticated study of how the meat in our skulls generates the self.}

I n my bolder moments, I consider neuroscience to be one of the most fundamental scientific fields. The brain is, after all, the location of our experiences and identities, and our main tool for understanding every facet of the Universe. The Brain by neuroscientist David Eagleman ambitiously promotes this view. Built around a series of fundamental questions, such as "what is reality?", it calls on a wide range of classic and recent findings, including innovative experiments by Eagleman himself, to demonstrate how brain science is optimally placed to answer those questions.

Eagleman begins by arguing that the brain determines who we are, and how we change. He illustrates just how dramatic such changes can be through the case of Charles Whitman, who in the 1960s switched from mild-mannered bank clerk to violent murderer because of a small tumour pressing on his amygdala, an area of the brain linked to aggression and fear.

Although the brain's development has a disproportionate role in human identity, with synaptic pruning in infancy a key shaping factor, our brains remain plastic throughout our lives. Eagleman demonstrates this with the well-known example of London taxi drivers found to have enlarged

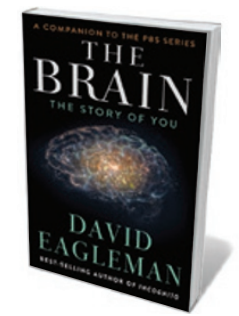

The Brain: The Story of You DAVID EAGLEMAN Pantheon: 2015. which false memories can be implanted. The emerging picture is far removed from one hippocampi - key to memory consolidation thousands of the capital's streets. Memory is the bedrock of our identities, but Eagleman highlights how the past is very much a reconstruction bordering on mythology. A case in point is the relative ease with - after memorizing

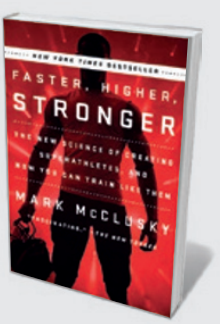

Faster, Higher, Stronger Mark McClusky (Plume, 2015)

From the primitive "bag-and-valve" apparatus used to measure runner's oxygen intake in the 1920 s to today's Silicon Valley performance labs, Mark McClusky shows how sports science has helped humans to push their physical limits, and why we keep striving to beat the best.

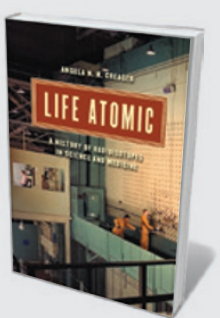


$\checkmark$ in which we have a single personal identity. Instead, he notes, "from cradle to grave, we are works in progress".

Everyone's world view, then, is fallible. Eagleman extends that idea by focusing on perception. We tend to assume that we have a direct connection to what is out there, and that all that we experience is all there is, but the truth is very different. He writes how "every sight, sound, smell - rather than being a direct experience, is an electrochemical rendition in a dark theater". But we are not experiencing a continuous flow of sight and sensation. We sample the world through saccades - jumping visual snapshots. From these, the brain constructs a continuous narrative heavily biased by expectations. We believe this narrative implicitly, even when it goes horribly wrong, such as in schizophrenic delusions.

In light of the idea that our view of our own minds is also deeply skewed, Eagleman challenges the primacy of consciousness. With most forms of expertise, such as driving, the conscious mind merely gets in the way. Furthermore, unconscious influences on our decisions are pervasive. For instance, judges deciding whether to award parole to prison inmates are more likely to do so if they have eaten beforehand. Decisions are little more than the product of unconscious neural battles between competing drives. Eagleman uses this stance to argue for an end to the catastrophic US war on drugs that began in the 1970s, and to call for more sympathy for, and (neuroscientific) understanding of, the plight of people addicted to drugs. He is helping addicts to reduce cravings by getting them to retrain their brain activity through neurofeedback in a functional magnetic resonance imaging scanner. They can view real-time summaries of the relative activities of their "craving" and "suppression" brain networks, and can practise strategies to discover the most effective way of suppressing cravings.

What of our relationships with others? Here, Eagleman notes that "what we demarcate as you is simply a network in a larger network". He describes how social exclusion can, like physical harm, activate pain centres in the brain such as the insula. Empathy,

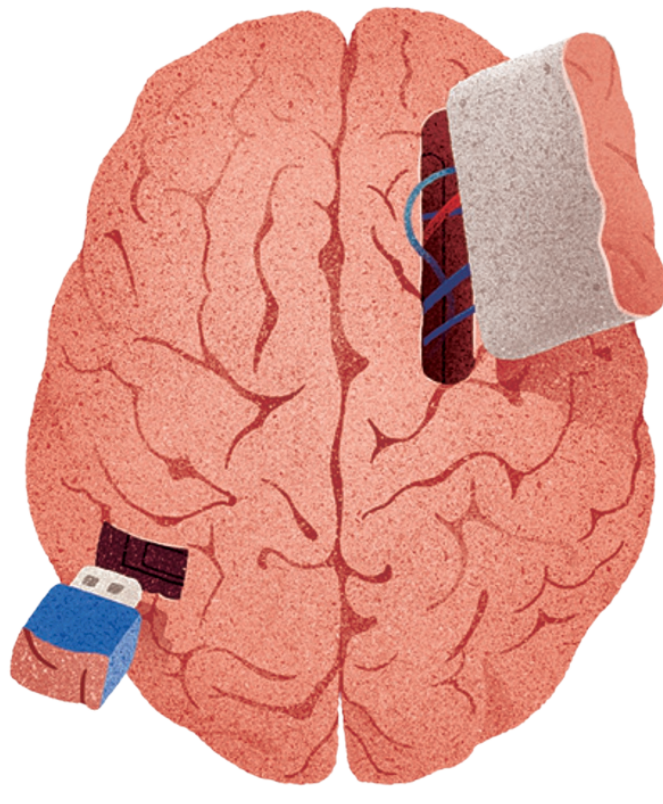

neurally speaking, invokes emotions as if we were experiencing for ourselves the events that we see others experience. However, when we consider members of "outgroups" to which we have no social ties, our empathic and social neural responses are flattened - as if we were dehumanizing them. This, Eagleman argues, is the neural mechanism that allows us to switch from being friendly to neighbours to wanting to wipe out their entire ethnic group, as for instance happened in the Bosnian war of the 1990s. Eagleman suggests that education about the neural underpinnings of our responses to outsiders is key to reducing the chance of genocides.

Eagleman ends by considering the future of humanity, and how neuroscience can technologically reshape almost every aspect of our lives. Although by far the most speculative part of the book, this is also the most fascinating. Eagleman describes the senses as flexible "peripheral plug-and-play" devices, with the brain not caring what input it receives as long as it is useful. We already exploit this feature with cochlear and retinal implants for people who have hearing or visual impairments. How much further can we take it?

Eagleman and his graduate student Scott Novich have developed an electronic vest that provides tactile feedback to the torso through arrays of small vibrating motors, and are testing it on people with impaired hearing, to allow them to 'hear' through touch.

The vest could be used for almost any real-time information stream, such as weather, stocks or altitude readouts in plane cockpits. And enhancing senses is only half the story: if we can control a robot arm through motor-cortex activity (see Nature 497, 176-178; 2013), could someone check their e-mails while their brain-computer interface manages a vacuum cleaner?

Throughout, Eagleman provides multiple, varied explanations for what consciousness is and what it is

for; he settles on neuroscientist Guilio Tononi's integrated information theory. This equates high levels of consciousness with information that is widespread throughout a network capable of supporting many different information states. Tononi's theory is consistent with the possibility of uploading our minds into computers. Without being limited by our fragile biology, we might feasibly travel to extrasolar worlds, "pausing" the computer simulation of our minds on the bulk of the journey to avoid boredom. Although such ideas are immensely fun to imagine, to computationally capture our brains we would have to be able to read every cellular detail of this incredibly complex organ - a feat that is centuries away, if it will ever be possible.

With such exciting themes, The Brain - a companion book to Eagleman's upcoming six-part television series of the same name on the US Public Broadcasting Service is an ideal introduction to how biology generates the mind. Readers familiar with this field will be revisiting a range of classic research, and might feel frustrated that more depth is not given in places. The science, however, is structured around crucial and wide-ranging questions, saturated with personal and social relevance. And Eagleman's answers are consistently clear, engaging and thought-provoking.

Daniel Bor is a neuroscientist at the University of Sussex in Brighton, UK, and is the author of The Ravenous Brain and co-author of 30-Second Brain. e-mail:danielxbor@gmail.com

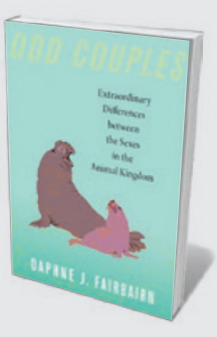

Odd Couples

Daphne J. Fairbairn (Princeton Univ. Press, 2015) As biologist Daphne Fairbairn observes, males and females of one species can differ greatly in colour, size and shape. Blanket-octopus females, for instance, outgrow males by 2 metres, quashing the dominant-male stereotype (see Suzanne Alonzo's review: Nature 496, 427-428; 2013).

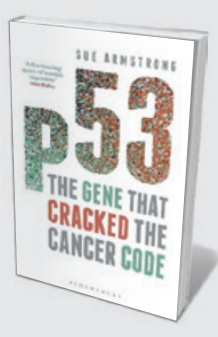

p53: The Gene that Cracked the Cancer Code Sue Armstrong (Bloomsbury Sigma, 2015) From its discovery in 1979 to its current place in cutting-edge gene therapy, p53 is the most studied gene in history. As Sue Armstrong details in this chronicle of genetics derring-do, its crucial role is to protect us from cancer, and the future of tumour treatment could depend on it. Emily Banham 